\title{
Época provável de maturação para diferentes cultivares de café arábica para o Estado de São Paulo
}

\author{
Estimated time of grain ripening of different arabica coffee cultivars for the São Paulo State, Brazil
}

\author{
Ludmila Bardin-Camparotto ${ }^{I}$ Marcelo Bento Paes de Camargo ${ }^{\text {II }}$ Jener Fernando Leite de Moraes ${ }^{\mathrm{II}}$
}

\section{RESUMO}

O sucesso da cafeicultura Paulista depende do conhecimento das condições climáticas que afetam a fenologia da planta, alterando a produtividade e a qualidade da produção. O fator térmico influi na duração da frutificação $e$ na época de maturação, ou seja, em regiões mais elevadas, com temperaturas mais amenas, a maturação ocorre mais lentamente. Nesse contexto, o objetivo deste trabalho foi gerar mapas de época provável de maturação de três diferentes cultivares de café arábica, baseados no acúmulo de graus-dia (GD), para o Estado de São Paulo, visando identificar regiões com diferentes potenciais para a qualidade de bebida, além de possibilitar a coleta de sementes antecipadamente, visando à formação de mudas para o início do período chuvoso. Os valores de GD foram acumulados a partir da data de floração por meio de SIG (sistemas de informações geográficas) até atingirem o total necessário de GD para as cultivares 'Mundo Novo', 'Catuaí' e 'Obatã'. A região centro oeste, devido a baixas altitudes (inferiores a 600m) e com elevadas temperaturas, apresentou o ciclo mais curto para as três cultivares, antecipando a maturação. Na região nordeste do Estado (Alta Mogiana), com altitudes superiores a 800m, a maturação ocorreu em períodos posteriores a maio, favorecendo a obtenção de bebidas de café com qualidade superior.

Palavras-chave: graus-dia, SIG, mapa, cafeicultura.

\section{ABSTRACT}

The successful development of the coffee crop in the State of Sao Paulo depends on the knowledge especially of climatic conditions that affect the plant phenology, that affect the productivity and the quality of crop production. The thermal factor influences the duration of fruiting and ripening stages.

\begin{abstract}
In regions with higher altitudes and milder air temperatures, the maturation of the coffee beans will happen more slowly. The objective was to generate maps of the probable time of ripening of three different cultivars based on accumulation of growing degree-days (GDD) for the São Paulo State. The maps can identify regions with different potential for quality coffee beverage. The monthly values of GDD were accrued, using the GIS. The maturation dates were estimated when the thermal sums of GDD were reached for each of the three cultivars, 'Mundo Novo', 'Catuaí' $e$ 'Obatã'. In the center-western region of the state due the low altitudes, below 600m, and the high temperatures, making the coffee grain ripening stage shorter for all the three cultivars. In the northeastern region of the state (Alta Mogiana) with altitudes more than $800 \mathrm{~m}$, ripening occurs after May. This climatic characteristics allow to obtain coffee beverage with superior quality.
\end{abstract}

Key words: degree-day, GIS, maps, coffee crop.

\section{INTRODUÇÃO}

Em São Paulo, existem áreas com agrossistemas de café que vêm se mantendo com destaque em relação às demais, devido principalmente às condições climáticas mais favoráveis ao cultivo do café. O café arábica apresenta ciclo fenológico com as fases de florescimento e maturação ocorrendo em épocas que variam em função das condições da região de cultivo. O fator térmico influi na duração da frutificação e na época de maturação; ou seja, quanto mais baixa a altitude e mais quente for a região, mais

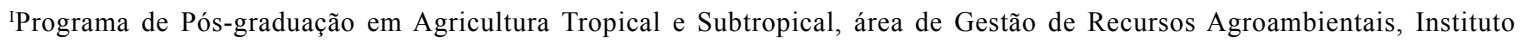
Agronômico (IAC), CP 28, 13001-970, Campinas, SP, Brasil. E-mail: ludmila_bardin@yahoo.com.br. Autor para correspondência. "Instituto Agronômico (IAC), Campinas, SP, Brasil. 
precoce será a maturação. Quando o ciclo da produção é muito curto ou é interrompido pela colheita, os ácidos clorogênicos fragmentados e o triptofano apresentam gosto amargo e adstringente, ocorrendo a formação da bebida com qualidade inferior. Porém, se o ciclo é longo, as transformações bioquímicas são completadas e ocorre acúmulo de precursores, levando o grão a apresentar características mais favoráveis de bebida (CORTEZ, 1997).

O cafeeiro arábica leva dois anos para completar o ciclo fenológico, sendo que a maturação plena ocorre quando pelo menos $50 \%$ dos grãos estão no estádio cereja, fato observado normalmente no Estado de São Paulo entre os meses de abril a junho (CAMARGO \& CAMARGO, 2001).

O desenvolvimento das espécies vegetais está associado a uma temperatura mínima necessária ao seu desenvolvimento, denominada temperaturabase $(\mathrm{Tb})$, e abaixo desta a planta não se desenvolve e se o fizer é em quantidade muito reduzida (PEREIRA et al., 2002). De acordo com CAMARGO (1974), cada espécie possui uma temperatura base, que pode variar de acordo com a fase fenológica da planta, entretanto, adota-se um valor médio para todo o ciclo da cultura, pela facilidade de aplicação.

Já o conceito de graus-dia (GD) assume a existência de uma temperatura basal abaixo da qual o crescimento vegetal pode ser desconsiderado. Cada grau acima da temperatura base corresponde a um graudia (PEZZOPANE et al., 2008). No caso do café, diversos trabalhos visando à maturação do fruto foram realizados utilizando a técnica de graus-dia. ORTOLANI et al. (2001) realizaram a regionalização da época de maturação do café arábica no Estado de São Paulo utilizando o acúmulo de graus-dia (floração-maturação) igual a $3500 \mathrm{GD}$ e temperatura-base igual a $10^{\circ} \mathrm{C}$.

Para o Estado do Paraná, PETEK et al. (2009) caracterizaram as exigências climáticas dos estádios fenológicos de cultivares de café arábica objetivando indicá-las para a tecnologia de escalonamento de colheita, para o Estado do Paraná. A soma térmica em graus-dia da florada até a maturação completa (cereja) foi de $2781 \pm 143,5 \mathrm{GD}$, considerando a Tb igual a $10,5^{\circ} \mathrm{C}$. Os autores observaram que o número de dias médio entre a florada e maturação, para o café arábica, foi de 210 a 250 dias. No Estado de Minas Gerais, LIMA \& SILVA (2008) determinaram as temperaturas base inferior e superior para o cafeeiro arábica na fase de implantação, sendo os valores obtidos 12,9 e $32,4{ }^{\circ} \mathrm{C}$, respectivamente.

PEZZOPANE et al. (2008) determinaram para o café arábica (cv. 'Mundo Novo') que a duração do período floração-colheita variou entre 202 a 250 dias em experimento realizado em Campinas, São Paulo, considerando o subperíodo (floração-colheita). Os valores de temperatura-base e soma térmica considerados foram de $10,2^{\circ} \mathrm{C}$ e 2887 graus-dia e $10,5^{\circ} \mathrm{C}$ e 2761 graus-dia, quando determinados sem e com correção pelo fator hídrico, respectivamente.

NUNES et al. (2010), baseados em dados fenológicos das cv. 'Mundo Novo', 'Catuaí' e 'Obatã' e de dados experimentais da região de Campinas e Mococa, estimaram a duração do estádio floraçãomaturação. Foi utilizado o valor de $\mathrm{Tb}=10,2^{\circ} \mathrm{C}$ (PEZZOPANE et al., 2008) e os valores de graus-dia obtidos foram: 2900 ('Mundo Novo'); 2990 ('Catuaí') e 3203 ('Obatã'). Os valores de duração do ciclo (floraçãomaturação) obtidos variaram entre 196 e 242 dias para a cv. 'Mundo Novo', resultado que está de acordo com os obtidos por PEZZOPANE et al. (2008). Para a cv. 'Catuaí', a duração do mesmo período variou entre 203 e 248 dias.

Considerando que o conhecimento das épocas prováveis de maturação do café arábica é fundamental para o planejamento agrícola, visando à previsão de safra, qualidade, indicação de origens e também comercialização, o objetivo do trabalho foi gerar mapas de épocas prováveis de maturação das cultivares 'Mundo Novo', 'Catuaí' e 'Obatã', com base em acúmulo de graus-dia. Este conhecimento poderá subsidiar a identificação de áreas com diferentes potenciais de qualidade natural de bebida de café no Estado de São Paulo, além de possibilitar a coleta de sementes antecipadamente, tendo em vista a formação de mudas para o início do período chuvoso.

\section{MATERIAL E MÉTODOS}

O período floração-maturação foi estimado segundo NUNES et al. (2010) que determinou as necessidades térmicas quantificadas pela acumulação de graus-dia (GD) para as cultivares de café 'Mundo Novo','Catuaí' e 'Obatã', as mais cultivadas no Estado de São Paulo. Os valores considerados para o complemento do período da floração-maturação foram: 2900GD para a cv. 'Mundo Novo'; 2990GD para cv. 'Catuaí'; e 3203GD para a cv. 'Obatã'. O cálculo do acúmulo de graus-dias (GD) foi determinado de acordo com a equação:

$$
\mathrm{GD}=\Sigma \text { Tmed }-\mathrm{Tb}
$$

em que Tmed $=$ temperatura média do ar $\left({ }^{\circ} \mathrm{C}\right)$ e Tb é a temperatura-base inferior $\left({ }^{\circ} \mathrm{C}\right)$, sendo o valor considerado para o período floração-maturação de $10,2^{\circ} \mathrm{C}$ (PEZZOPANE et al., 2008). 
A época de ocorrência de florada considerada no trabalho foi determinada por ZACHARIAS et al. (2008), que observou que a época mais provável de ocorrência de florada no Estado de São Paulo é no segundo decêndio de setembro; razão pela qual a data da florada considerada neste trabalho foi 15 de setembro.

Os mapas de valores acumulados de GD, que mostram a estimativa da época de maturação das cultivares 'Mundo Novo', 'Catuaí' e 'Obatã', foram elaborados utilizando o modelo numérico digital das temperaturas médias mensais (mapas mensais). As temperaturas médias mensais foram obtidas por meio de equações utilizando a altitude e a latitude como variáveis independentes e a temperatura do ar como variável dependente, de acordo com o modelo linear simples:

$$
Y=a+b x+c x_{1}
$$

em que $Y=$ temperatura máxima ou mínima média mensal ou anual $\left({ }^{\circ} \mathrm{C}\right)$; a e b são os coeficientes da equação de regressão; x é a altitude (metros); e $\mathrm{x}_{1}$ é a latitude (minutos). As equações de regressão desenvolvidas, o coeficiente de determinação $\left(R^{2}\right)$ e a significância de cada fator das equações de ajuste foram avaliados pelo teste $\mathrm{F}$ ao nível de significância de $5 \%$.

As equações de regressão desenvolvidas e o modelo numérico do terreno (SRTM, 2006) foram processados em ambiente SIG (sistema de informações geográficas), programa ILWIS (Fonte: http:// www.itc.nl), gerando assim um modelo numérico dos valores de temperatura média do ar. Os resultados foram utilizados como base no cálculo e na geração de mapas de época de maturação a partir de modelos de grausdia acumulados.

Os valores de GD foram acumulados mês a mês, utilizando o SIG-ILWIS, de acordo com BARDIN et al. (2010b), considerando a florada em 15 de setembro: 'Mundo Novo': (2900-(((gdset/30)*15)+(gdout+ gdnov + gddez + gdjan + gdfev + gdmar $))) /($ gdabr2/30); 'Catuaí': (2990-(((gdset/30)*15)+(gdout+gdnov + gddez+gdjan+gdfev+gdmar $))) /($ gdabr/30); 'Obatã': (3203-((gdset/30)*15+(gdout + gdnov + gddez + gdjan + gdfev + gdmar + gdabr $))) /($ gdmai/31); em que: gdjan = mapa mensal de GD acumulado do mês de janeiro; gdfev = mapa mensal de GD acumulado do mês de fevereiro; gdmar = mapa mensal de GD acumulado do mês de março; gdabr = mapa mensal de GD acumulado do mês de abr; gdmai = mapa mensal de GD acumulado do mês de mai; gdjun = mapa mensal de GD acumulado do mês de junho; gdjul = mapa mensal de GD acumulado do mês de julho; gdago = mapa mensal de GD acumulado do mês de agosto; gdset = mapa mensal de GD acumulado do mês de setembro; gdout = mapa mensal de GD acumulado do mês de outubro; gdnov = mapa mensal de GD acumulado do mês de novembro; gddez = mapa mensal de GD acumulado do mês de dezembro.

A data de maturação foi considerada, quando o valor total de GD foi atingido, tendo sido obtida pela divisão do total de graus-dia do mês de maturação (último mês da equação) pelo número de dias do mês, obtendo-se assim valores diários de grausdia. A partir desse ponto, os valores diários de grausdia foram somados até atingirem 2900GD ('Mundo Novo'), 2990GD ('Catuaí') e 3203GD ('Obatã'), determinando a data estimada de maturação.

\section{RESULTADOS E DISCUSSÃO}

$\mathrm{Na}$ tabela 1, são apresentados os coeficientes de regressão múltipla e os coeficientes de determinação $\left(\mathrm{R}^{2}\right)$ para estimativa das temperaturas médias do ar, em função da altitude e da latitude. Observou-se que todas as regressões foram significativas ao nível de $5 \%$ de probabilidade. Os valores de $\mathrm{R}^{2}$ também foram elevados, variando entre 0,89 (junho e julho) a 0,96 (outubro). Valores de $R^{2}$ menores nos meses de inverno foram também observados por outros autores, PINTO \& ALFONSI (1974); PEDRO JR. et al. (1991); BARDIN et al. (2010a).

O Estado de São Paulo apresenta grande diversidade de regimes térmicos que causam variações significativas no ciclo das culturas. Visando ao auxílio na identificação de áreas com diferentes ciclos e tipos de qualidade natural do café arábica, no Estado de São Paulo, foram gerados mapas da época provável de maturação do café arábica (Figura 1) para as cvs. 'Mundo Novo' (A), 'Catuaí' (B) e 'Obatã' (C). De maneira geral, observa-se que regiões com temperaturas mais amenas e altitudes mais elevadas apresentaram maior duração do ciclo (período entre floração e maturação plena), como podemos observar principalmente na região nordeste (Alta Mogiana).

De maneira geral, para a cv. 'Mundo Novo', as regiões Noroeste, Araraquarense, Nova Alta Paulista e Marília apresentam datas de maturação anteriores a 15 de março. Fato que pode ser explicado pelas temperaturas mais elevadas encontradas nessas regiões, tornando o ciclo do café mais curto e consequentemente antecipando a colheita, o que poderá favorecer o desenvolvimento de processos fermentativos deletérios à qualidade da bebida 
Tabela 1 - Coeficientes das equações de regressão múltipla e coeficientes de determinação $\left(\mathrm{R}^{2}\right)$ para estimativa das temperaturas médias mensais e anual, em função da altitude e latitude para o Estado de São Paulo.

\begin{tabular}{|c|c|c|c|c|}
\hline Mês & Constante & Coeficiente angular da altitude & Coeficiente angular da latitude & $\mathrm{R}^{2}$ \\
\hline Jan & 32,23 & $-0,0070^{*}$ & $-0,0027^{*}$ & $0,95^{*}$ \\
\hline Fev & 32,62 & $-0,0076^{*}$ & $-0,0026^{*}$ & $0,95^{*}$ \\
\hline Mar & 34,17 & $-0,0075^{*}$ & $-0,0041^{*}$ & $0,95^{*}$ \\
\hline Abr & 35,15 & $-0,0076^{*}$ & $-0,0063^{*}$ & $0,95^{*}$ \\
\hline Maio & 37,99 & $-0,0069^{*}$ & $-0,0106^{*}$ & $0,94 *$ \\
\hline Jun & 38,18 & $-0,0069^{*}$ & $-0,0118^{*}$ & $0,89 *$ \\
\hline Jul & 37,31 & $-0,0079^{*}$ & $-0,0107^{*}$ & $0,89^{*}$ \\
\hline Ago & 42,71 & $-0,0079 *$ & $-0,0135^{*}$ & $0,90 *$ \\
\hline Set & 44,31 & $-0,0075^{*}$ & $-0,0139 *$ & $0,91 *$ \\
\hline Out & 44,97 & $-0,0078^{*}$ & $-0,0132 *$ & $0,96 *$ \\
\hline Nov & 41,02 & $-0,0079^{*}$ & $-0,0097 *$ & $0,95^{*}$ \\
\hline Dez & 37,22 & $-0,0080^{*}$ & $-0,0065^{*}$ & $0,94 *$ \\
\hline Anual & 38,10 & $-0,0075^{*}$ & $-0,0088^{*}$ & $0,95^{*}$ \\
\hline
\end{tabular}

$*=$ significativo a $5 \%$ de probabilidade.

(CORTEZ, 1997). O mesmo fato pode ser observado para a maturação dos frutos nas outras duas cvs. ('Catuaí' e 'Obatã'), já que a colheita também é antecipada nessas regiões. Já na região leste-nordeste do Estado, em regiões com maiores altitudes e temperaturas mais amenas, a maturação ocorre em períodos posteriores a maio, prolongando o ciclo da cultura.

Observou-se a duração do ciclo para alguns municípios. Para a cv. 'Mundo Novo', nos municípios de Franca e Pedregulho (Alta Mogiana), o ciclo estimado foi de 284 e 288 dias, respectivamente. Já na região oeste do Estado, onde as temperaturas são mais elevadas e a altitude menor, o município de Andradina teve seu ciclo estimado em 197 dias; Adamantina em 211 dias; e Presidente Prudente em 213 dias.

Acv. 'Catuaí' apresentou valores de duração do ciclo próximos aos da cv. 'Mundo Novo', em função do número de GD necessários para completar seu ciclo variar em apenas 90 GD. O município de Pedregulho apresentou a maior diferença, sendo de 15 dias a mais que para a cv. 'Mundo Novo' (303 dias). Em média, a diferença entre a duração de ciclo das duas cultivares foi de oito dias, variando de 6 a 15 dias. As maiores diferenças ocorreram em regiões de maior altitude, onde o ciclo é mais longo, como em Pedregulho (15 dias), Franca (12 dias) e Bragança Paulista (12 dias). Para regiões mais quentes, como Andradina, Adamantina e Presidente Prudente, a variação foi em torno de sete dias, sendo a duração do ciclo da cv. 'Catuaí' para esses municípios igual a 204, 218 e 220 dias, respectivamente.
A cv. 'Obatã', mais tardia, apresentou a duração do ciclo entre 197 (Ubatuba) e 338 (Pedregulho). O município de Franca teve o ciclo estimado em 330 dias, Campinas 290 dias e Ribeirão Preto 249 dias. Regiões com maior altitude apresentaram as maiores diferenças entre o comprimento dos ciclos entre as cvs 'Mundo Novo' e 'Obatã', de 50 dias em Pedregulho, 49 dias em Bragança Paulista e 46 dias em Franca. As regiões de menor altitude, mais quentes, situadas no centro-oeste do Estado, apresentaram diferenças entre 20 a 30 dias, como Marília (26 dias) e Dracena (22 dias). Adiversidade de altitudes, de condições climáticas e das exigências das três cultivares condicionam uma grande variação das épocas da maturação no Estado de São Paulo. Dessa forma, a qualidade natural da bebida do café é dependente destes fatores.

\section{CONCLUSÃO}

No Estado de São Paulo, onde as temperaturas são mais elevadas (região centro-oeste), a maturação do café arábica ocorre com até três meses de antecedência, comparada à região nordeste (Alta Mogiana), possibilitando a coleta de sementes antecipadamente, visando à formação de mudas para $\mathrm{o}$ início do período chuvoso. A Alta Mogiana, com altitudes entre 800 a $1200 \mathrm{~m}$, apresenta condição climática propícia à maturação dos frutos e consequentemente com potencial para obtenção de qualidade natural superior de bebida. 


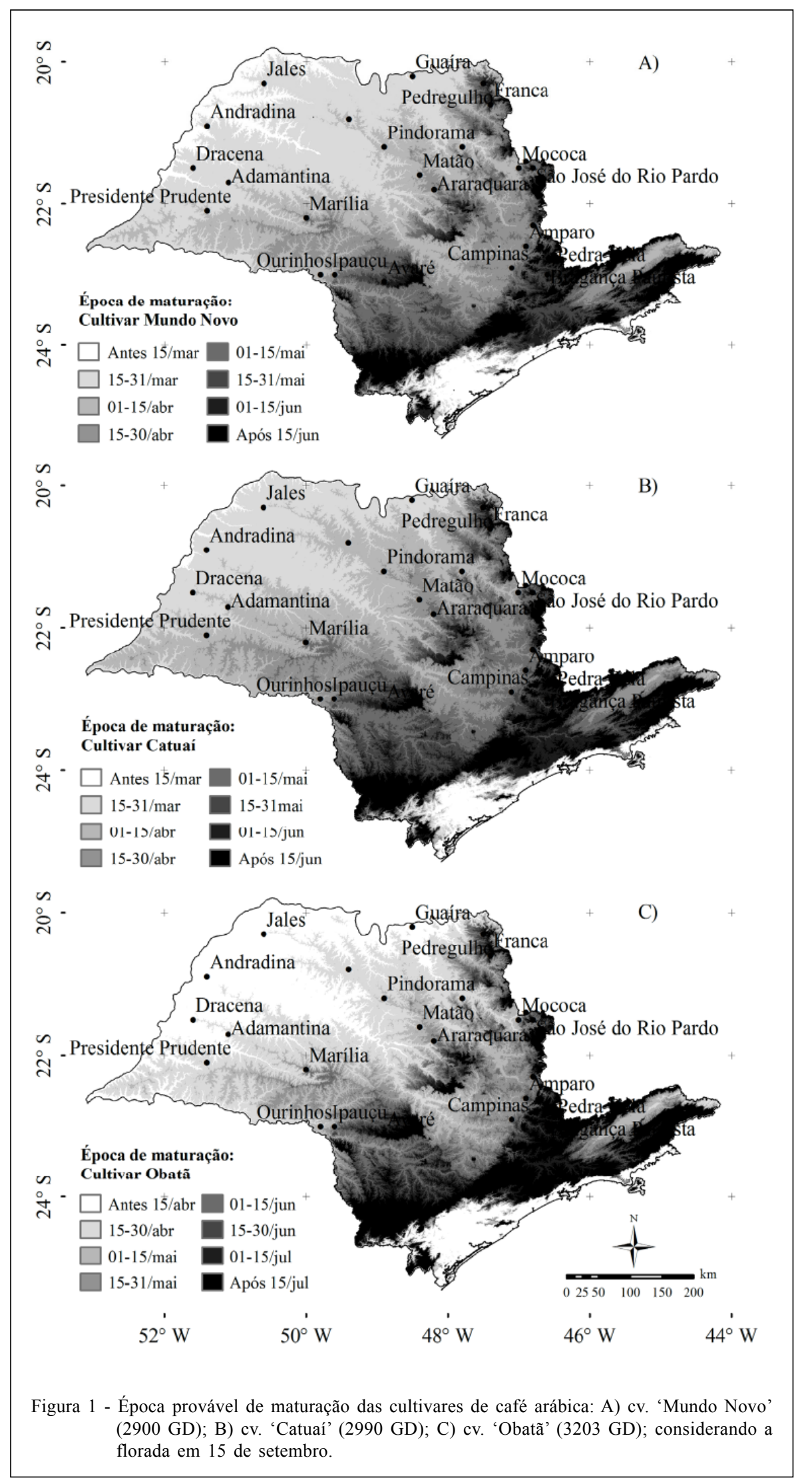

Ciência Rural, v.42, n.4, abr, 2012. 


\section{AGRADECIMENTO}

À Fundação de Amparo à Pesquisa do Estado de São Paulo (FAPESP), pela concessão da bolsa de Pós-graduação (Doutorado) para Ludmila Bardin-Camparotto. Ao Conselho Nacional de Desenvolvimento Científico e Tecnológico (CNPq), pela concessão de bolsa ao autor Marcelo Bento Paes de Camargo.

\section{REFERÊNCIAS}

BARDIN, L. et al. Estimativa das temperaturas máximas e mínimas do ar para a região do Circuito das Frutas, SP. Revista Brasileira de Engenharia Agrícola e Ambiental, v.14, p.618-624, 2010a. Disponível em: <http://www.scielo.br/ s c i e lo.ph p s c ript=s ci_arttext \& pid = S 1415 $43662010000600008 \& \operatorname{lng}=\mathrm{en} \& \mathrm{nrm}=\mathrm{iso}>$. Acesso em: 15 set. 2011. doi: 10.1590/S1415-43662010000600008.

BARDIN, L. et al. Estimativas das épocas de colheita da videira "Niagara Rosada" na região do Pólo Turístico do Circuito das Frutas, São Paulo. Scientia Agraria, v.11, p.135-139, 2010 b.

CAMARGO, A.P. Clima. In: Cultura de café no Brasil. Rio de Janeiro: Instituto Brasileiro do Café, 1974 p. $20-35$.

CAMARGO, A.P.; CAMARGO, M.B.P. Definição e esquematização das fases fenológicas do cafeeiro arábica nas condições tropicais do Brasil. Bragantia, v.60, p.65-68, 2001. Disponivel em: <http:/www.scielo.br/scielo.php?pid=S0006$87052001000100008 \&$ script $=$ sci arttext $>$. Acesso em: 24 ago. 2011. doi: 10.1590/S0006-87052001000100007.

CORTEZ, J.G. Aptidão climática para qualidade de bebida nas principais regiões cafeeiras de Minas Gerais. Informe Agropecuário, v.18, p.27-31, 1997.

LIMA, E.; SILVA, E.L. Temperatura base, coeficientes de cultura e graus-dia para cafeeiro arábica em fase de implantação. Revista Brasileira de Engenharia Agrícola e Ambiental v.12, n.3, p.266-273, 2008. Disponível em: <http:// www.scielo.br/scielo.php?script $=$ sci_arttext\&pid $=\mathrm{S} 1415$ 43662008000300007>. Acesso em: 24 ago. 2011. doi: $10.1590 / \mathrm{S} 1415-43662008000300007$.

NUNES, F.L. et al. Modelos agrometeorológicos de estimativa da duração de estádio floração-maturação para três cultivares de café arábica. Bragantia, v.69, p.1011-1018, 2010. Disponível em: <http:/www.scielo.br/scielo.php?pid=S000687052010000400029\&script $=$ sci_arttext $>$. Acesso em: 15 jan. 2011. doi: 10.1590/ S0006-87052010000400029.

ORTOLANI, A.A. et al. Regionalização da época de maturação e qualidade natural de bebida do café arábica no Estado de São Paulo. In: CONGRESSO BRASILEIRO DE AGROMETEOROLOGIA, 12., 2001, Fortaleza, CE. Anais... Fortaleza: SBA, 2001. V.1, p.53-54.

PEDRO JÚNIOR, M.J. et al. Estimativa das temperaturas médias mensais das máximas e das mínimas no estado de São Paulo. Campinas : Instituto Agronômico de Campinas, 1991. 11p. (Boletim Técnico, 142).

PEREIRA, A.R. et al. Agrometeorologia: fundamentos e aplicações práticas. Piracicaba: Agropecuária, 2002. 478p.

PETEK, M.R. et al. Exigências climáticas para o desenvolvimento e maturação dos frutos de cultivares de coffea arábica. Bragantia, v.68, n.1, p.169-181, 2009. Disponível em: <http://www.scielo.br/ s c i e lo.php? s cript=s ci arttext \& pid = S 0006 $87052009000100018 \& \operatorname{lng}=\mathrm{en} \& n \mathrm{~nm}=\mathrm{iso}>$. Acesso em: 20 maio, 2011. doi: 10.1590/ S0006-87052009000100018.

PEZZOPANE, J.R.M. et al. Exigência térmica do café arábica Mundo Novo no subperíodo florecimento-colheita. Ciência e Agrotecnologia, v.32, n.6, p.1781-1786, 2008. Disponível em: $<$ http://www.scielo.br/scielo.php? pid=S 1413 $70542008000600016 \&$ script $=\mathrm{sci}$ abstract\&tlng $=\mathrm{pt}>$. Acesso em: 23 ago. 2011. doi: 10.1590/ S1413-70542008000600016.

PINTO, H.S.; ALFONSI, R.R. Estimativa das temperaturas médias, máximas e mínimas mensais no Estado do Paraná, em função de altitude e latitude. São Paulo: Universidade de São Paulo, 1974. 28p. (Caderno de Ciências da Terra, 52).

SRTM. Shuttle Radar Topography Mission. Acesso em: 29 ago. 2006. Online. Disponível em: <http://srtm.usgs.gov>.

ZACHARIAS, A.O. et al. Modelo agrometeorológico de estimativa do início da florada plena do cafeeiro. Bragantia, n.67, p.249-256, 2008. Disponível em: $<$ http://www.scielo.br/scielo.php?pid=S0006$87052008000100030 \&$ script $=$ sci arttext $>$. Acesso em: 15 mar. 2010. doi: 10.1590/ S0006-87052008000100030. 


\section{ERRATA}

Artigo originalmente publicado sem a equação $Y=a+b x+c x_{1}$ na página 595

\section{Época provável de maturação para diferentes cultivares de café arábica para o Estado de São Paulo}

Estimated time of grain ripening of different arabica coffee cultivars for the São Paulo State, Brazil

Ludmila Bardin-Camparotto ${ }^{\mathrm{I}}$ Marcelo Bento Paes de Camargo ${ }^{\mathrm{II}}$ Jener Fernando Leite de Moraes ${ }^{\mathrm{II}}$

Para html ou PDF, acesse:

http://www.scielo.br/scielo.php?script=sci_arttext\&pid=S0103-84782012000400003\&lng=pt\&nrm=iso\&tlng=pt

ou

http://www.scielo.br/pdf/cr/v42n4/a9612cr5899.pdf

IPrograma de Pós-graduação em Agricultura Tropical e Subtropical, área de Gestão de Recursos Agroambientais, Instituto Agronômico (IAC), CP 28, 13001-970, Campinas, SP, Brasil. E-mail: ludmila_bardin@yahoo.com.br. Autor para correspondência. "Instituto Agronômico (IAC), Campinas, SP, Brasil. 